\title{
Electrochemistry in Micro- and Nanochannels Controlled by Streaming Potentials
}

\author{
Zinaida A. Kostiuchenko, Jin Z. Cui, and Serge G. Lemay*
}

Cite This: J. Phys. Chem. C 2020, 124, 2656-2663

Read Online

ABSTRACT: Fluid and charge transport in micro- and nanoscale fluidic systems are intrinsically coupled via electrokinetic phenomena. While electroosmotic flows and streaming potentials are well understood for externally imposed stimuli, charge injection at electrodes localized inside fluidic systems via electrochemical processes remains to a large degree unexplored. Here, we employ ultramicroelectrodes and nanogap electrodes to study the subtle interplay between ohmic drops, streaming currents, and faradaic processes in miniaturized channels at low concentrations of supporting electrolyte. We show that electroosmosis can, under
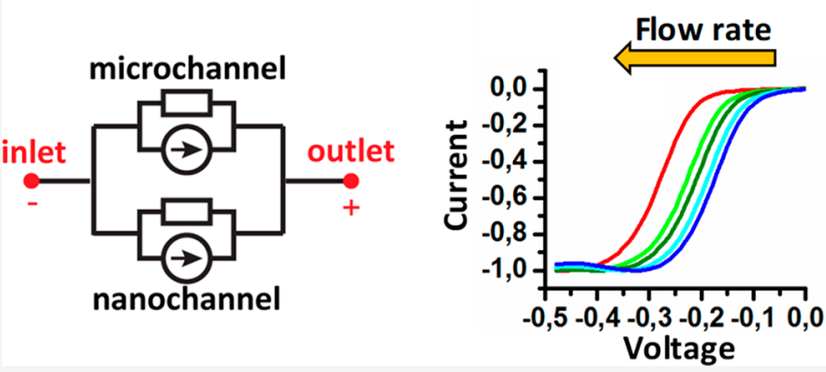
favorable circumstances, counteract the effect of ohmic losses and shift the apparent formal potential of redox reactions. This interplay can be described by simple circuit models, such that the results described here can be adapted to other micro- and nanofluidic electrochemical systems.

\section{INTRODUCTION}

Electrochemical methods provide one of the most direct and easily implemented routes for detection in lab-on-a-chip analysis systems. Mass transport of redox-active analytes in confined geometries may however be strongly influenced by factors not encountered in macroscopic systems. In particular, migration and convection become intrinsically coupled due to the presence of the electrical double layer (EDL) at the solidliquid interface. This can result in electroosmotic flows, streaming currents, and enhanced surface conduction. ${ }^{1}$ In addition, ohmic potential drops are more pronounced due to the high resistances inherent to narrow channel geometries. Electrochemical measurements conventionally rely on an excess of inert supporting electrolyte to provide high solution conductivity, which also tends to minimize the impact of electrokinetic effects. In samples where the ionic strength is inherently low, however, this simplification breaks down and the influence of confinement on mass transport is enhanced. ${ }^{2-5}$ Studies of mass transport and electron transfer kinetics for redox-active species under low salt conditions for microelectrodes, ${ }^{6}$ nanopore electrode arrays, ${ }^{7,8}$ and thin-layer cells $^{9-11}$ explicitly show that electrostatics affect both the magnitude of redox currents and the shape of voltammetric responses. The corresponding impact of electrokinetic effects has received limited attention, notable exceptions being studies of power generation ${ }^{12-14}$ and electrokinetically driven bipolar electrodes. ${ }^{15}$ Our focus here is on electrodes imbedded inside confined fluidic circuits, as commonly occur with meso- or nanoporous electrodes and in $\mu$ TAS applications.

Streaming effects are caused by the convective transport of ions in the EDL. A pressure-driven flow carries with it the uncompensated charge in the EDL and thus generates a streaming current. This current can be measured by providing a low-resistance electrical pathway in parallel to the fluidic system, as sketched in Figure 1a. In many fluidic systems, however, this electrical pathway is absent. Charge then accumulates at the inlets and outlets of channels, or at junctions between channels with different geometries, causing electric fields to develop. These electric fields generate socalled backflow currents that oppose the streaming currents. In the steady state, this results in streaming potentials across channels that are proportional to the flow rate of fluid through the channel, as sketched in Figure 1b. Importantly, the occurrence of faradaic processes inside the fluidic system can lead to behavior that is intermediate between the limiting cases of Figure la,b.

The magnitude of the streaming current (Figure 1a) for laminar flow in a cylindrical capillary with a radius much larger than the Debye screening length is ${ }^{16}$

$$
i_{\text {str }}=\frac{8 \varepsilon_{0} \varepsilon \zeta}{R^{2}} Q
$$

Here, $R$ is the radius of the channel, $\zeta$ is the $\zeta$ potential of the channel walls, $\varepsilon_{0} \varepsilon$ is the permittivity of the solution, and $Q$ is the volumetric flow rate. The corresponding expression for

Received: September 10, 2019

Revised: December 20, 2019

Published: January 9, 2020 


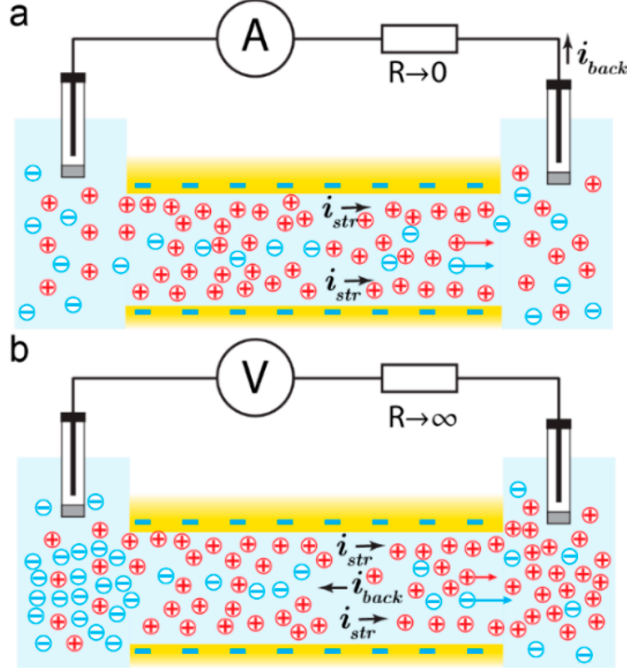

Figure 1. Schematic illustration of streaming effects in response to a pressure-driven flow in a channel with negatively charged walls. (a) If an external low-resistance electrical pathway exists, here represented by two reference electrodes and an ammeter, an uncompensated streaming current $i_{\text {str }}$ flows along the channel. (b) If no external pathway exists, charge accumulates at the inlet and outlet and a streaming potential develops across the channel that can be measured externally. The streaming potential drives a reverse migration current through the channel (the backflow current, $i_{\text {back }}$ ) that cancels the streaming current. More complex situations arise when electrodes are present in the channel since streaming potentials can drive electrochemical reactions while streaming currents can compensate for ohmic drops by carrying (parts of) the faradaic currents.

the streaming potential in the absence of an external pathway for the current (Figure $1 b$ ) is

$$
V_{\text {str }}=\frac{8 \varepsilon_{0} \varepsilon \zeta L}{\pi K R^{4}} Q
$$

where $\mathrm{K}$ is the conductivity of the solution and $L$ is the length of the channel. Both $i_{\text {str }}$ and $V_{\text {str }}$ are proportional to the volumetric flow rate $Q$. Equation 2 indicates that the magnitude of the streaming potential is proportional to the $\zeta$ potential and inversely proportional to the conductivity of the medium. Dilution of the supporting electrolyte leads to an increase in $\zeta$ potential ${ }^{17,18}$ and a decrease of the solution conductivity, both of which promote the buildup of streaming potentials and thus strengthen the possible impact of convection on electrochemical reactions taking place at electrodes inside the channel. The inverse power dependence on $R$ in eq 1 and eq 2 means that for a given flow rate $Q$ streaming effects become increasingly relevant with decreasing channel cross-section.

Here, we focus on electrochemical reactions at multiple electrodes imbedded in micro- and nanofluidic channels under pressure-driven flow conditions. We show that electrokinetic effects influence faradaic processes at individual electrodes and provide a coupling mechanism between electrodes localized at different positions in a fluidic system. This behavior is captured by a simple equivalent circuit model that can easily be adapted to other fluidic circuits.

\section{MATERIALS AND EXPERIMENTAL METHODS}

System Geometry. We focus on two lithographically fabricated electrochemical systems sketched in Figure 2a. The
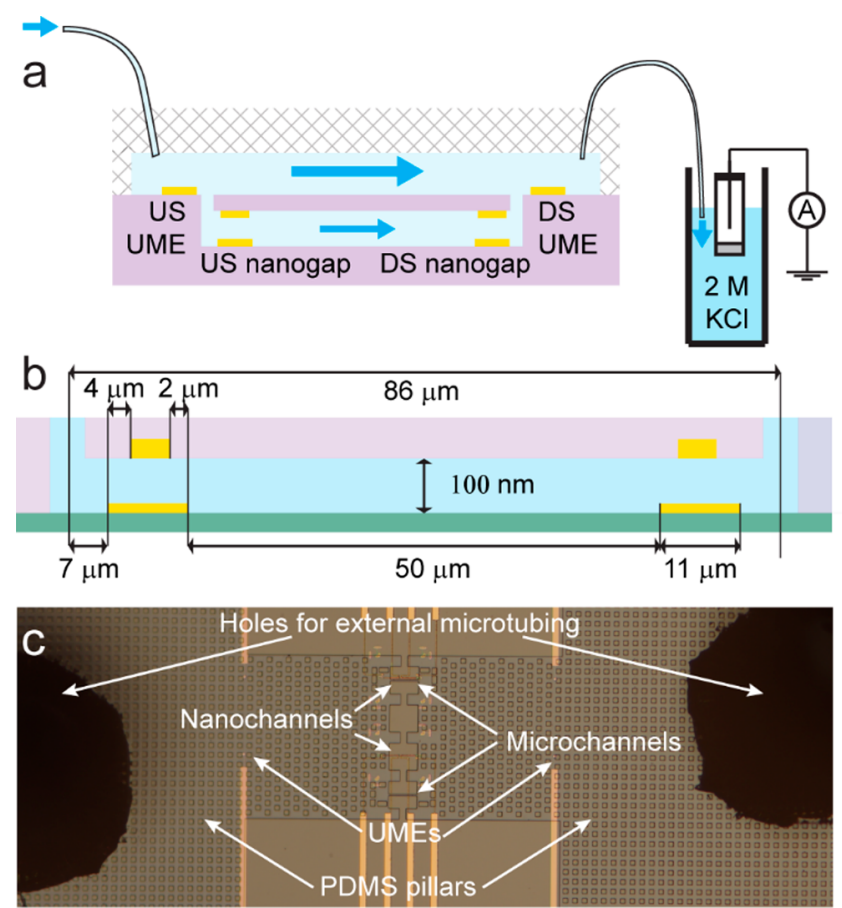

Figure 2. Experimental configuration. (a) Schematic side view of fluidic channels with measurement electrodes. In the UME configuration, two disk electrodes are located in reservoirs at either end of the microchannel and the nanochannel is absent. In the nanogap configuration, a nanofluidic channel containing two nanogap devices is connected in parallel with the microfluidic channel. In both cases, fluid is introduced at constant flow rate (blue arrows) and extracted into an external reservoir in which a reference electrode is immersed. (b) Dimensions of the nanochannel and nanogap device. (c) Micrograph of the complete system showing the relative position of the UMEs, the microchannels, and the nanochannels. The regular array of squares consists of PDMS pillars that support the inlet and outlet reservoirs.

UME configuration consists of two individually addressable ultramicroelectrodes ( $5 \mu \mathrm{m}$ radius Pt disks, UMEs) located in two large reservoirs linked by microchannels. The nanogap configuration consists of a nanochannel with two nanogap devices ${ }^{19}$ embedded along its length (dimensions are given in Figure $2 b$ ). The nanochannel is connected in parallel with a microchannel identical to that of the UME configuration. Each nanogap device consist of two planar electrodes positioned in the floor and ceiling of the nanochannel. When suitable overpotentials are applied to the two electrodes, redox cycling takes place and leads to a highly amplified, steady-state faradaic current flowing from one electrode to the other. ${ }^{19}$

For both configurations, the microfluidic layer is implemented by bonding a polydimethylsiloxane (PDMS) block to the chip bearing the electrodes, as shown in Figure $2 \mathrm{c}$. The microfluidic circuit consists of two parallel microchannels (length $90 \mu \mathrm{m}$, width $5 \mu \mathrm{m}$ wide and height $5 \mu \mathrm{m}$ ) linking two wide access regions supported by pillars. Fluid access is provided by punching holes through the PDMS block and inserting polytetrafluoroethylene (PFTE) microtubes.

Device Fabrication. The electrodes and nanofluidic channels were formed using lithography-based microfabrication techniques, as described in detail in the Supporting Information. In short, a thermally oxidized silicon wafer was used as substrate. The UMEs were patterned from an e-beam evaporated Pt film by optical lithography and lift-off. The 
nanogap devices were created by the same process through subsequent deposition and patterning of the $\mathrm{Pt}$ bottom electrode, a $\mathrm{Cr}$ sacrificial layer defining the nanogap, and a Pt top electrode. The wafer was then passivated with a dielectric layer $\left(\mathrm{SiO}_{2}\right.$ or $\left.\mathrm{SiN}\right)$ to prevent contact between the metal and liquid. Finally, access of the electrodes was provided by etching openings in the passivation layer using reactive ion etching (RIE). To define the microchannels, a microstructure was formed on the bottom of a PDMS block by curing a PDMS layer on top of a lithographically fabricated SU-8 mold. The PDMS inlet and outlet reservoirs were connected using polytetrafluoroethylene (PFTE) microtubes with an inner diameter of $0.3 \mathrm{~mm}$.

Chemicals. We focus on reversible, outer sphere redox couples. The analytes $1,1^{\prime}$-ferrocenedimethanol $\left(\mathrm{Fc}(\mathrm{MeOH})_{2}\right)$ and hexaamineruthenium(III) chloride $\left(\mathrm{Ru}\left(\mathrm{NH}_{3}\right)_{6} \mathrm{Cl}_{3}\right)$, and the supporting electrolyte salt (potassium chloride BioUltra for molecular biology $\geq 99.5 \%, \mathrm{KCl}$ ) were purchased from SigmaAldrich and used as received. The solutions were prepared with Milli-Q water of $18.2 \mathrm{M} \Omega \cdot \mathrm{cm}$ resistivity. The solutions were equilibrated with ambient atmosphere over the course of the measurements and therefore had a $\mathrm{pH}$ near 5.6. Selectipur chromium etchant for releasing the sacrificial layer was obtained from BASF.

Instrumentation. The electrostatic potentials of all active working electrodes were set with respect to an $\mathrm{Ag} / \mathrm{AgCl}$ reference electrode (MF 2079, RE-5B, BaSi) and the corresponding currents measured via inverting transimpedance amplifiers (Femto DDCPA-300) operated as sourcemeters. No potentiostatic feedback or auxiliary electrode were needed as the current through the reference electrode was less than $1 \mathrm{nA}$ throughout.

Experimental Protocol. Equivalent devices were used for all measurements. For experiments with UMEs, the $\mathrm{Cr}$ sacrificial layer in the nanochannels was left unetched, thus excluding the nanochannels from consideration. For experiments with nanogap devices, the nanochannel was released by exposure to a droplet of $\mathrm{Cr}$ etchant, following which the chip was flushed with Milli-Q water and dried.

The PDMS block containing the microfluidic structure was then bonded to the chip. To do so, we first activated the surfaces of the PDMS and chip in oxygen plasma. The separate components were then aligned and contacted under a microscope and placed in an oven for $15 \mathrm{~min}$ at $70{ }^{\circ} \mathrm{C}$ to strengthen the bonding.

Fluid was supplied to the inlet tube using a $500 \mu \mathrm{L}$ ILjS microsyringe driven by a syringe pump (Pump 11 Pico Plus Elite, Harvard) at constant flow rate. The end of the fluid outlet was immersed in a beaker containing $2 \mathrm{M} \mathrm{KCl}$ aqueous solution, and the $\mathrm{Ag} / \mathrm{AgCl}$ reference electrode was placed in this reservoir to establish the reference potential. The UME or nanogap device that was closest to the inlet from the syringe pump was named "upstream" (US), and that which was closest to the reference electrode was named "downstream" (DS) .

Prior to the experiments, the electrodes were cleaned by repeated voltammetric cycling in $0.5 \mathrm{M}$ sulfuric acid until the cyclic voltammograms (CV) became reproducible. A syringe with the test solution was then connected to the inlet. Experiments were started with the lowest concentration of supporting electrolyte $(10 \mu \mathrm{M} \mathrm{KCl})$ and made to proceed in consecutive 10 -fold increments of the salt concentration in order to prevent residual salt from being trapped in the PDMS.

\section{THEORETICAL MODEL}

For a reversible reaction with fast electron transfer kinetics, the current $i$ at a UME is given by the expression

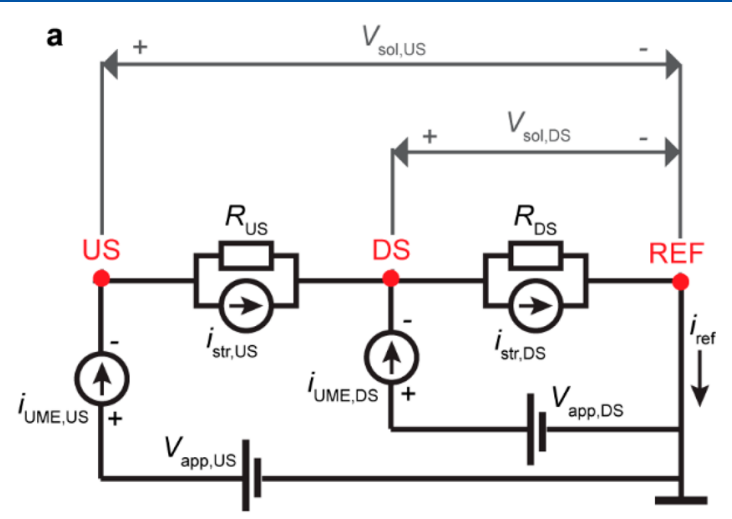

b

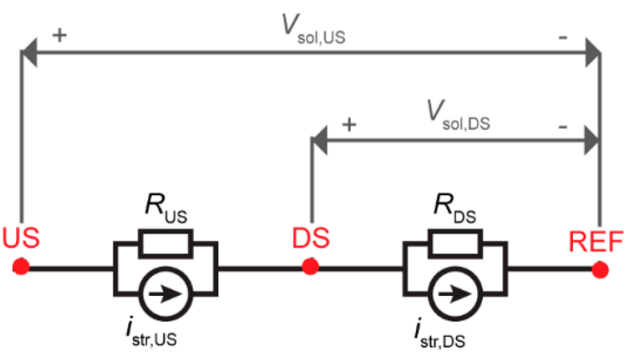

Figure 3. (a) Equivalent electrical circuit for the UME configuration including fluidic components $\left(R_{\mathrm{US}}, R_{\mathrm{DS}}, i_{\mathrm{str}, \mathrm{US}}\right.$, and $\left.i_{\mathrm{str}, \mathrm{DS}}\right)$, reactions at the electrodes $\left(i_{\mathrm{UME}, \mathrm{US}}\right.$ and $i_{\mathrm{UME}, \mathrm{DS}}$, measured $)$ and the external circuit $\left(V_{\text {app,US }}, V_{\text {app,DS }}\right.$, and $\left.i_{\text {ref }}\right)$. The red labels represent the positions of the US electrode, the DS electrode, and the reference electrode. (b) Equivalent electrical circuit for the nanogap configuration.

$$
\begin{aligned}
i= & \frac{i_{\text {lim }, \text { an }}}{1+\exp \left\{-(F / R T)\left(V_{\text {app }}-V_{\text {sol }}-E_{0}\right)\right\}} \\
& +\frac{i_{\text {lim }, \mathrm{cat}}}{1+\exp \left\{(F / R T)\left(V_{\mathrm{app}}-V_{\mathrm{sol}}-E_{0}\right)\right\}}
\end{aligned}
$$

Here, $i_{\text {lim,an }}$ is the anodic limiting (mass-transport-limited) current, $i_{\text {lim,cat }}$ is the cathodic limiting current, $F$ is the Faraday constant, $R$ is the gas constant, $T$ is the absolute temperature, $E_{0}$ is the reaction formal potential, $V_{\text {app }}$ is the potential applied to the electrode, and $V_{\text {sol }}$ is the solution potential at the electrode. All potentials are referred to the reference electrode.

In a conventional electrochemical cell with a high conductivity electrolyte, $V_{\text {sol }} \approx 0$. This assumption however breaks down if the solution resistance is high and faradaic processes cause ohmic drops to develop. Micro/nanofluidic systems are particularly prone to ohmic drops because of their confined geometry and small dimensions. Streaming potentials can also lead to $V_{\text {sol }} \neq 0$, which in our experimental configuration can occur, to varying degrees, in the microchannels, nanochannels, outlet reservoirs, and outlet microtube.

UME Configuration. Figure $3 \mathrm{a}$ shows an equivalent electrical circuit for the UME system in which the different components are represented by lumped circuit elements. We concentrate on the steady state, hence capacitive elements such as the EDL capacitance of the electrodes are omitted. Ground represents the reference electrode potential. The solution 

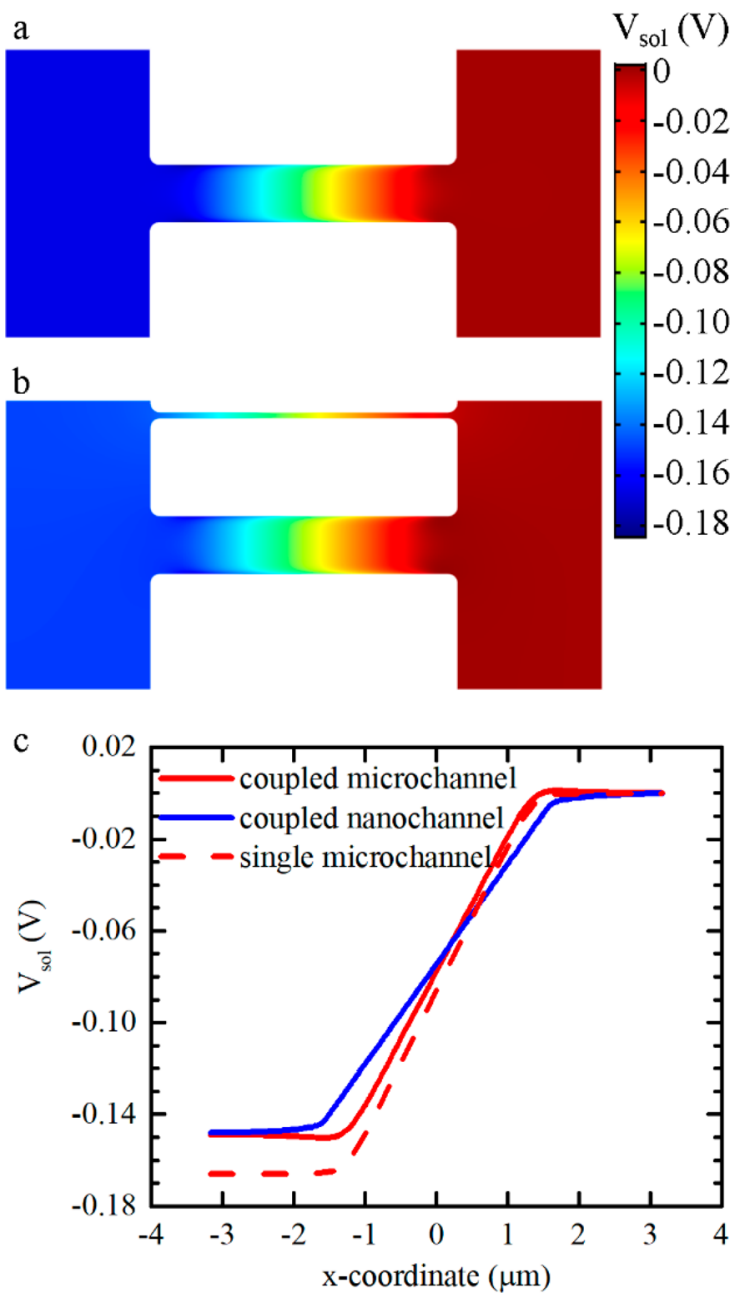

Figure 4. Simulation illustrating the interplay between streaming effects in two channels connected in a parallel flow configuration. To improve readability, the dimensions employed here differ from those of the actual device but the same behavior is observed in both cases. (a) Solution potential $V_{\text {sol }}$ versus position in a single microchannel (length $3 \mu \mathrm{m}$, height $600 \mathrm{~nm}$ ) with negatively charged walls. A streaming potential develops between the inlet and the outlet. (b) The same microchannel in parallel with a nanochannel (height 30 $\mathrm{nm}$ ) with neutral walls. The microchannel dominates the electrokinetic response and imposes a streaming potential similar to that in part a, as discussed in the text. This causes an electric field and accompanying backflow current in the nanochannel even though no streaming current is induced in this channel. (c) Solution potential versus position along the central axes of the channels (dashed red line, microchannel of panel a; solid red line, microchannel of panel b; solid blue line, nanochannel). The potential gradient in the nanochannel is very close to linear. Details of the simulation are given in the Supporting Information.

potentials at the two electrodes are $V_{\text {sol, US }}$ and $V_{\text {sol,Ds}} \cdot R_{\mathrm{DS}}$ and $i_{\text {str,DS }}$ represent, respectively, the (ionic) resistance and the streaming current through the channels downstream of the DS electrode. For simplicity, the pillar region and its connecting microtube are lumped together in the DS circuit elements. Similarly, $R_{\mathrm{US}}$ and $i_{\text {str,US }}$ represent the resistance and (flow-rate controlled) streaming current in the region between the two UMEs. The faradaic processes taking place at the two UMEs are represented by voltage-controlled current sources, $i_{\mathrm{UME}, \mathrm{DS}}$ and $i_{\mathrm{UME}, \mathrm{Us}}$. The currents are specified by eq 3 in which the positive and negative terminals in the circuit diagram play the role of $V_{\text {app }}$ and $V_{\text {sol }}$, respectively. In the experiments, $i_{\text {ref }}$ as well as $i_{\mathrm{UME}, \mathrm{DS}}$ or $i_{\mathrm{UME}, \mathrm{US}}$ are measured. Finally, we ignore the inlet channel. Since no electrical connection exists in this pathway, it cannot generate currents or streaming potentials that influence $V_{\text {sol,us }}$ and $V_{\text {sol,Ds. }}$.

The equivalent circuit of Figure $3 \mathrm{a}$ indicates that there exists a subtle interplay between faradaic processes and streaming currents. First, consider the case where a faradaic reaction takes place at the DS electrode $\left(i_{\mathrm{UME}, \mathrm{DS}} \neq 0\right)$ and the US part of the circuit is ignored. In the absence of fluid flow $\left(i_{\text {str,US }}=i_{\text {str,DS }}=\right.$ 0 ), the solution potential at the DS electrode is given by Ohm's law, $V_{\text {sol,DS }}=R_{\mathrm{DS}} i_{\mathrm{UME}, \mathrm{DS}}$. This ohmic drop diminishes the interfacial potential difference and hinders the reaction as is commonly the case. In the presence of fluid flow $\left(i_{\text {str,DS }}>0\right)$, charge conservation at the DS node dictates that $V_{\text {sol,DS }}=$ $R_{\mathrm{DS}}\left(i_{\mathrm{UME}, \mathrm{DS}}-i_{\text {str,DS }}\right)$. This result shows how the streaming current can diminish or even reverse the sign of the solution potential (or, from a different viewpoint, the streaming current can carry part or all of the electrochemically generated current, mitigating ohmic drops). If the US components are also considered, current flows become more complex since faradaic currents injected at either electrode can influence both $V_{\text {sol,us }}$ and $V_{\text {sol,Ds. }}$ In terms of the experimentally observable currents, the contributions to the observed solution potential shifts $V_{\text {sol,DS }}$ and $V_{\text {sol,Us }}$ from ohmic contributions are

$$
\begin{aligned}
& V_{\mathrm{ohm}, \mathrm{DS}}=R_{\mathrm{DS}} i_{\mathrm{REF}} \\
& V_{\mathrm{ohm}, \mathrm{US}}=R_{\mathrm{DS}} i_{\mathrm{REF}}+R_{U S} i_{\mathrm{UME}, \mathrm{US}}
\end{aligned}
$$

while those from streaming potentials are

$$
\begin{aligned}
& V_{\mathrm{str}, \mathrm{DS}}=-R_{\mathrm{DS}} i_{\mathrm{str}, \mathrm{DS}}=V_{\mathrm{sol}, \mathrm{DS}}-V_{\mathrm{ohm}, \mathrm{DS}} \\
& V_{\mathrm{str}, \mathrm{US}}=-R_{\mathrm{DS}} i_{\mathrm{str}, \mathrm{DS}}-R_{\mathrm{US}} i_{\mathrm{str}, \mathrm{US}}=V_{\mathrm{sol}, \mathrm{US}}-V_{\mathrm{ohm}, \mathrm{US}}
\end{aligned}
$$

as derived in the Supporting Information.

Faradaic currents at electrodes imbedded in fluidic channels can also generate electroosmotic flows (EOF). This represents a form of coupling between faradaic reactions and convection that is complementary to streaming effects. While interesting for future work, this factor did not play a role in the experiments presented here as they were performed at a constant volumetric flow rate. The effect is therefore excluded from the equivalent circuit model.

Nanogap Configuration. The equivalent circuit model needs to be adjusted in two ways to describe the experiments using a parallel nanochannel containing US and DS nanogap devices.

First, no UMEs are present. Therefore, in terms of the equivalent circuit, we have $i_{\mathrm{UME}, \mathrm{US}}=i_{\mathrm{UME}, \mathrm{DS}}=0$. Simultaneously, no net current is generated by the nanogap devices. Electroactive species that are reduced or oxidized at the top electrode are converted back to their original state at the bottom electrode via redox cycling. Since the bottom electrode extends further US and DS compared to the top electrode, as shown in Figure 2b, the collection efficiency is essentially 100\% and no charge is injected into the nanochannel beyond the nanogap devices. ${ }^{20}$ The absence of current sources greatly simplifies the equivalent circuit of Figure $3 a$ to that of Figure 3b.

Second, both the nano- and microchannels carry streaming currents. In practice, however, the microchannel dominates and largely determines the streaming potential difference between the inlet and outlet of the nanochannel. Qualitatively, 


\section{upstream UME}

a

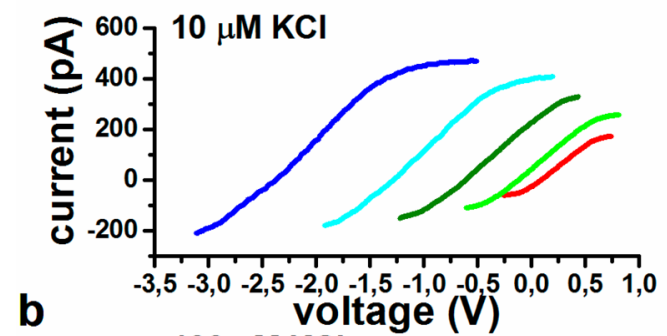

b

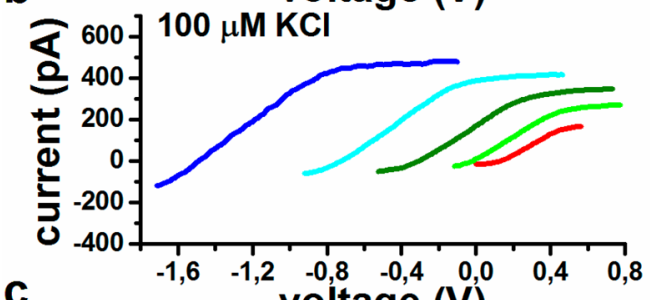

C

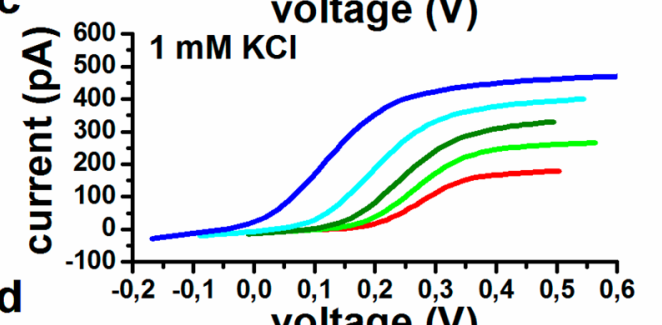

d

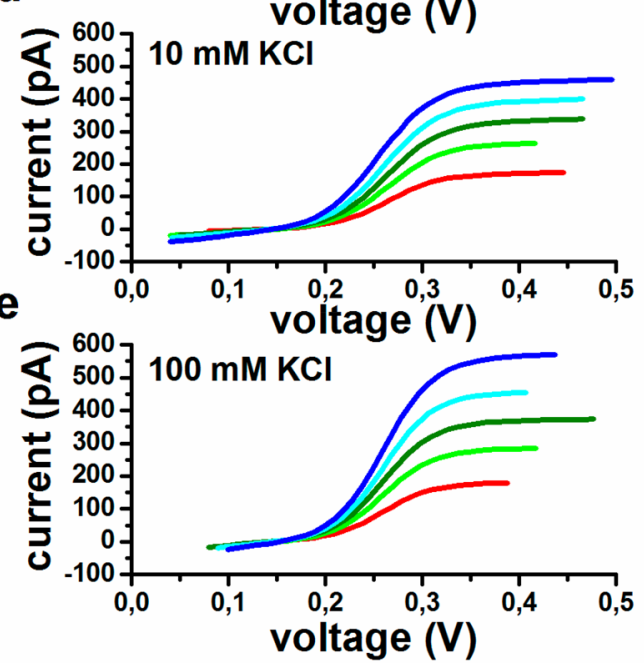

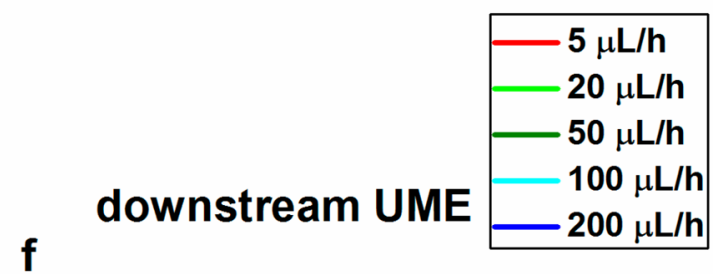

f

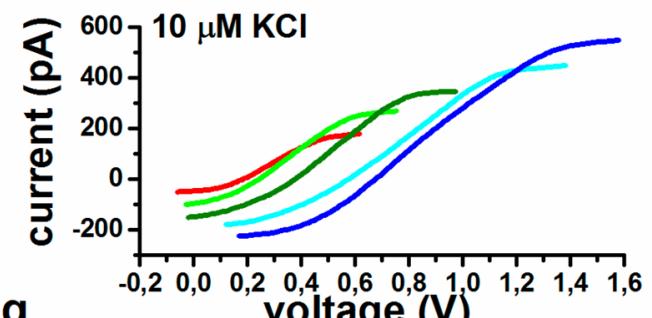

9

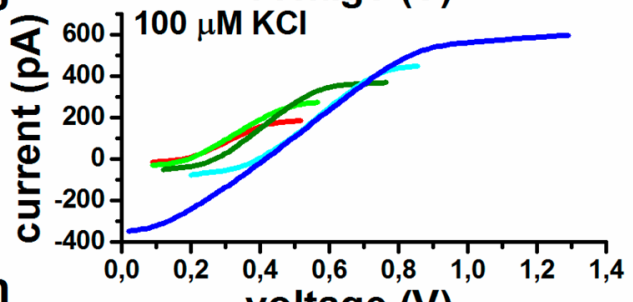

h
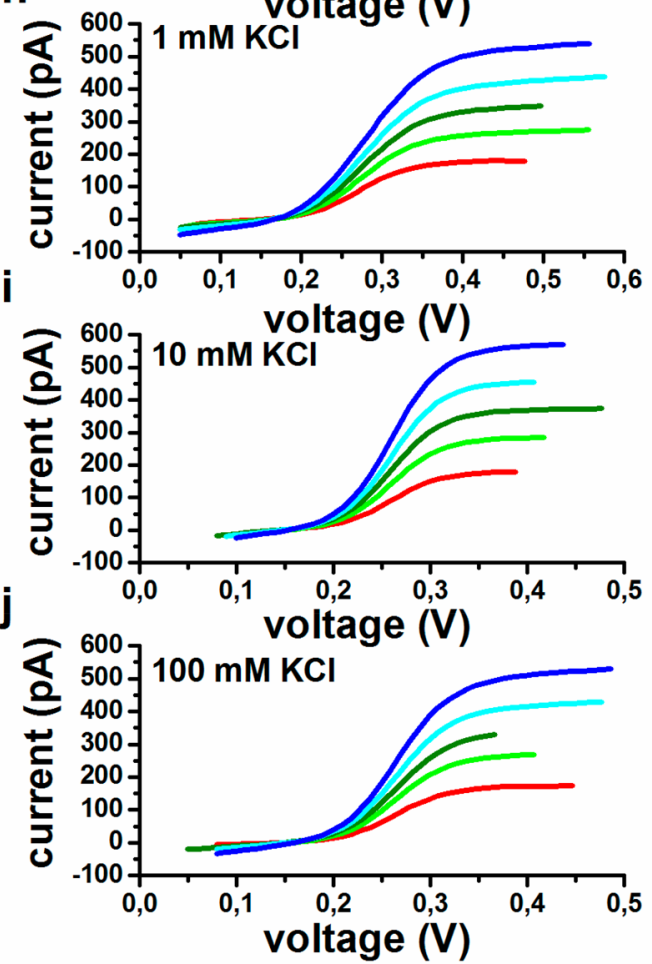

Figure 5. Cyclic voltammetry in the UME configuration. The US (parts a-e) and DS (parts $\mathrm{f}-\mathrm{j}$ ) UMEs were separately cycled at different flow rates for $100 \mu \mathrm{M} \mathrm{Fc}(\mathrm{MeOH})_{2}$ in $\mathrm{KCl}$ solutions of $10 \mu \mathrm{M}(\mathrm{a}, \mathrm{f}), 100 \mu \mathrm{M}(\mathrm{b}, \mathrm{g}), 1 \mathrm{mM}(\mathrm{c}, \mathrm{h}), 10 \mathrm{mM}(\mathrm{d}, \mathrm{i})$, and $100 \mathrm{mM}(\mathrm{e}, \mathrm{j})$. In each case, the electrode not being cycled was poised at $0 \mathrm{~V}$.

this is easy to understand. The net backflow resistance is determined by the resistances of the two channels in parallel, which is mostly set by the much smaller resistance of the microchannel. This is discussed quantitatively in the Supporting Information (eq S8) and illustrated qualitatively via finite-element simulations in Figure 4. The simulation shows two channels with a large difference in cross-section and different surface charges (Figure $4 \mathrm{~b}$ ). The net streaming potential is close to that expected for the larger channel alone shown in Figure 4a (more specifically, the streaming potential is $\sim 9 \%$ smaller in Figure $4 \mathrm{~b}$, consistent with the decrease in backflow resistance as expected from eq S8). This streaming potential difference causes a linear potential gradient along the length of the smaller channel, which can influence faradaic reactions taking place in nanogap devices inside that channel (Figure 4c). 
The nanogap devices are located next to the inlet and outlet of the nanochannel such that, to a good approximation, they correspond to the US and DS nodes in Figure 3. In the more general case, electrodes could be located deeper inside the channel. In this case, however, the streaming potential remains linear with the flow rate. The streaming currents, $i_{\text {str,Us }}$ and $i_{\text {str,DS}}$, can then be seen as effective values that parametrize the shifts in $V_{\text {sol,Us }}$ and $V_{\text {sol,DS }}$ due to streaming potentials.

\section{RESULTS AND DISCUSSION}

UME Configuration. Convection strongly affects the rate of mass transport to the UMEs. The values of $i_{\text {lim,an }}$ and $i_{\text {lim,cat }}$

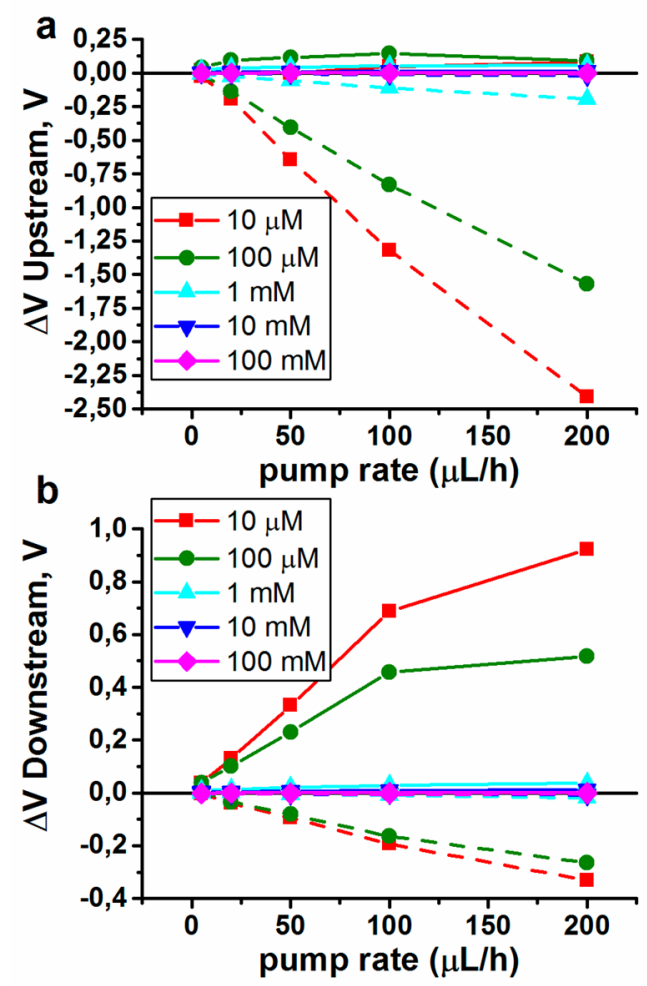

Figure 6. Contributions to the solution potential from ohmic drops ( $V_{\text {ohm,US }}$ and $V_{\text {ohm,DS }}$, solid lines) and streaming potentials $\left(V_{\text {str,US }}\right.$ and $V_{\text {str,DS}}$, dashed lines) associated with (a) the US UME and (b) the DS $\mathrm{UME}$ for $\mathrm{KCl}$ concentrations of $10 \mu \mathrm{M}, 100 \mu \mathrm{M}, 1 \mathrm{mM}, 10 \mathrm{mM}$, and $100 \mathrm{mM}$. In each case, the second UME is kept at $0 \mathrm{~V}$.

therefore depend on the flow rate. For experiments with UMEs, we used ferrocene dimethanol $\left(\mathrm{Fc}(\mathrm{MeOH})_{2}\right)$ as analyte. The reduced form of $\mathrm{Fc}(\mathrm{MeOH})_{2}$ is neutral and its mass transport is therefore unaffected by electric fields even at low supporting electrolyte concentrations. We first performed cyclic voltammetry at the upstream electrode with the downstream electrode poised at $0 \mathrm{~V}$, then switched the role of the electrodes. We performed each measurement at pump rates of $200,100,50,20$, and $5 \mu \mathrm{L} / \mathrm{h}$. A zero flow rate was not employed because the complete interruption of liquid injection would leave diffusion as the only mechanism for mass transport to the UMEs. Because of the quasi-two-dimensional geometry, this does not lead to a well-defined steady-state current.

Figure 5 shows CVs obtained for salt concentrations in the range of $10 \mu \mathrm{M}$ to $100 \mathrm{mM}$. The experimental curves at high salt concentrations exhibit sigmoidal shapes with well-defined plateaus and a half-wave potential of $\sim 0.26 \mathrm{~V}$ for all flow rates,

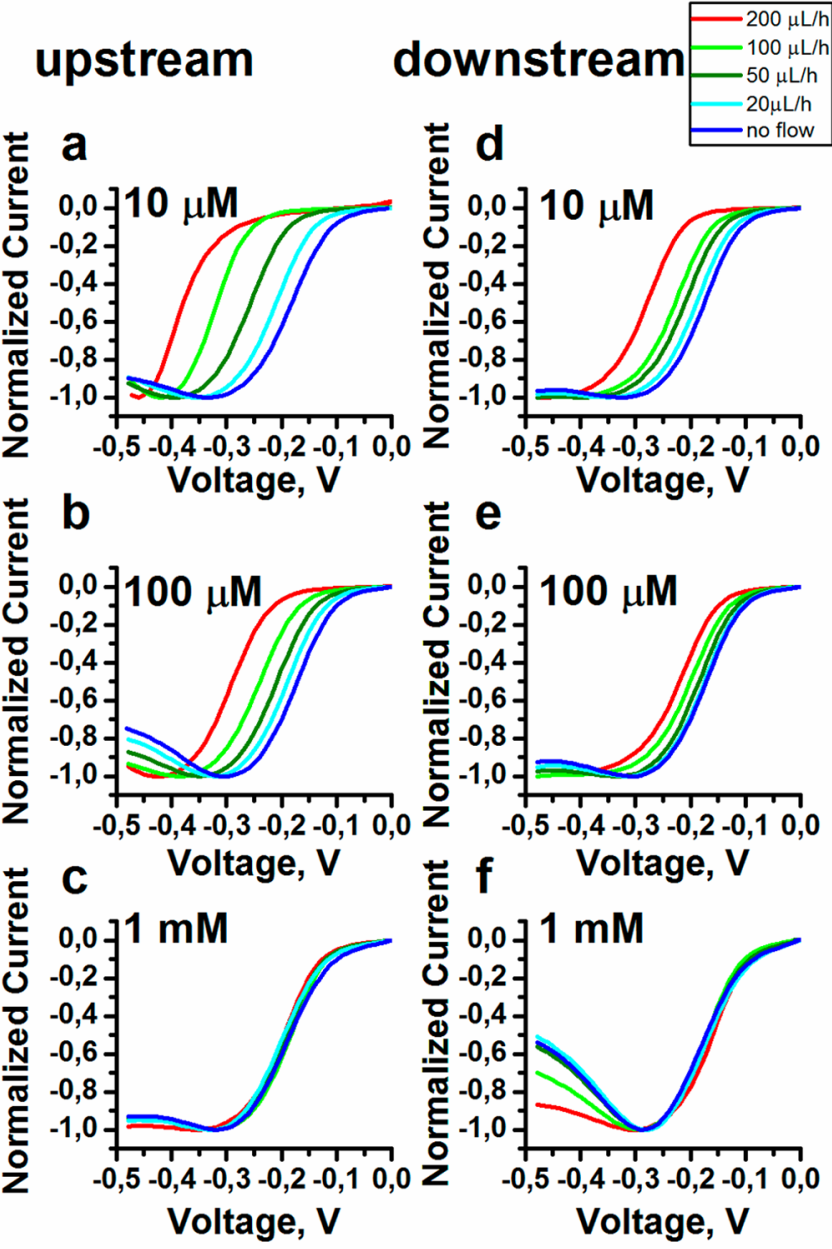

Figure 7. Cyclic voltammetrics for $100 \mu \mathrm{M} \mathrm{Ru}\left(\mathrm{NH}_{3}\right)_{6} \mathrm{Cl}_{3}$ in $\mathrm{KCl}$ solutions of $10 \mu \mathrm{M}(\mathrm{a}, \mathrm{d}), 100 \mu \mathrm{M}(\mathrm{b}, \mathrm{e}), 1 \mathrm{mM}(\mathrm{c}, \mathrm{f})$ for the upstream (parts $a-c)$ and downstream (parts $d-f$ ) nanogap devices.

as expected. The magnitude of the mass-transport-limited current increases with increasing flow rate due to convection. For solutions with low ionic strengths, the voltammograms become stretched and exhibit a potential shift that depends on the flow rate. Strikingly, the direction of the potential shift is opposite for the two electrodes: while curves at the US move toward more negative (reducing) potentials with increasing flow rate, those at the DS move toward more positive (oxidizing) potentials. At $10 \mu \mathrm{M}$ salt concentration, the potential shift is so pronounced that flow can induce oxidation to take place at highly reducing electrode potentials of ca. $-2 \mathrm{~V}$ for the US electrode. In this case, the free energy to drive the faradaic reaction is provided mechanically via the syringe pump, as previously reported for bipolar electrodes in microchannels. ${ }^{15}$

In order to interpret these results, we applied the model of Figure 3 (eqs $4 \mathrm{a}$ and $4 \mathrm{~b}$ and eq 5) to extract values for the ohmic drops and streaming currents, as summarized in Figure 6. The solid lines represent $V_{\text {ohm,US }}$ and $V_{\text {ohm,DS }}$, the ohmic potential drops that would be generated in the absence of streaming currents. These have a positive sign, and the effect is stronger for the downstream UME (when the US UME is cycled, the grounded DS UME acts as a counter electrode and absorbs part of the current, decreasing the ohmic drop). The dashed lines represent the streaming potentials, $V_{\text {str,Us }}$ and 

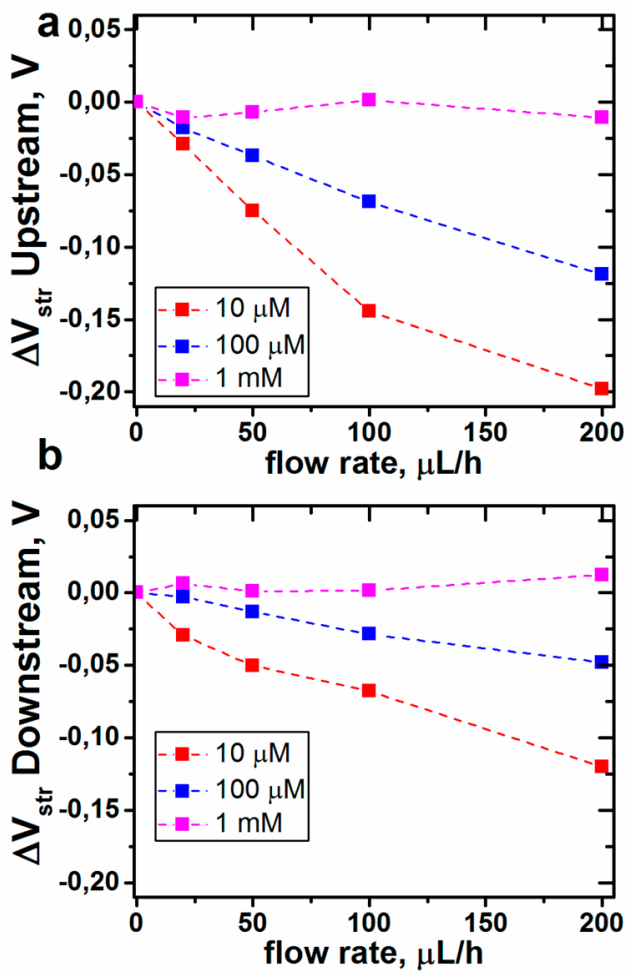

Figure 8. Values of the streaming potentials in the fluidic system associated with the nanogap devices (a) upstream and (b) downstream respect with the channels region for the $\mathrm{KCl}$ concentrations $10 \mu \mathrm{M}, 100 \mu \mathrm{M}$, and $1 \mathrm{mM}$.

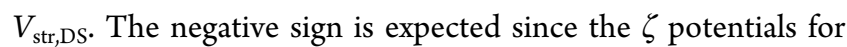
all materials in the system $\left(\mathrm{SiO}_{2}, \mathrm{PDMS}\right.$, PTFE) are negative and both $i_{\text {str,US }}$ and $i_{\text {str,DS }}$ are thus positive. The magnitude of the streaming potentials increases approximately linearly with flow rate and decreases with increasing salt concentration, as expected. Finally, the magnitude of the streaming potential is higher for the US UME than for the DS UME. This is also expected based on eqs $4 \mathrm{a}$ and $4 \mathrm{~b}$ and eq 5: $V_{\text {str,Ds }}$ is generated by $i_{\text {str,DS }}$ only, while $V_{\text {str,Us }}$ has contributions from both $i_{\text {str,DS }}$ and $i_{\text {str,Us. }}$.

The streaming potentials developed in the microfluidic system make the solution potential more negative and enhance the interfacial potential difference at the US UME. This shifts the voltammogram toward negative (reducing) potentials, as seen in Figure 5 $(\mathrm{a}-\mathrm{e})$. Simultaneously, ohmic drops shift the potentials required to drive reactions to toward positive (oxidizing) values. At the DS electrode, the streaming potential is much lower and the ohmic drop term prevails, as seen in Figure $5(\mathrm{f}-\mathrm{j})$. The dependence of $V_{\text {ohm, US }}$ and $V_{\text {ohm,DS }}$ on $V_{\text {app,US }}$ and $V_{\text {app,DS }}$ is also less linear than that on $V_{\text {str,Us }}$ and $V_{\text {str,DS}}$, as expected, since the former depend in a complex manner on the potential-dependent faradaic currents while the latter depending only on the flow rate $Q$.

Redox Cycling in Nanogap Devices. For experiments involving nanofluidic channels, we used ruthenium hexamine, $\mathrm{Ru}\left(\mathrm{NH}_{3}\right)_{6}{ }^{3+}$, as analyte because the redox cycling voltammograms for this species exhibit a more reversible shape and a higher limiting current under low salt conditions compared to ferrocene dimethanol. ${ }^{11}$ The magnitude of the redox cycling current is set by the local concentration of redox species inside the nanogap and is largely independent of convective mass transport of either the reduced or oxidized forms of the analyte. $^{21}$ We performed every measurement for the pump rates $200,100,50,20 \mu \mathrm{L} / \mathrm{h}$ and in the absence of flow. The hydraulic resistances of each of the micro- and nanochannels were $4.3 \times 10^{15}$ and $2.0 \times 10^{20} \mathrm{~Pa} \cdot \mathrm{s} / \mathrm{m}^{3}$, respectively, as estimated from their geometry and assuming laminar flow. ${ }^{22} \mathrm{~A}$ pump rate of $200 \mu \mathrm{L} / \mathrm{h}$ thus corresponds to a pressure of 1.2 bar across the parallel channels and an average fluid velocity of $1200 \mu \mathrm{m} / \mathrm{s}$ in the nanochannel. We were able to suspend flow entirely here ( $0 \mu \mathrm{L} / \mathrm{h}$ pump rate) because, as discussed in the theory section, the nanogap device "recycles" molecules in the detection region and a true steady state can be achieved even in a confined channel geometry. We first cycled the top electrode of the upstream nanogap device and then the top electrode of the downstream device. All noncycled electrodes were kept at a potential of $0 \mathrm{~V}$ with respect to the $\mathrm{Ag} / \mathrm{AgCl}$ reference electrode.

Figure 7 shows a set of redox cycling curves obtained with the same nanofluidic channel at three salt concentrations. The plots in the left and right columns correspond to the US and DS nanogap devices, respectively. We normalize the CVs to their maximum value to facilitate the visualization and extraction of changes in the value of the half-wave potential. At both the upstream and downstream nanogaps, clear shifts are observed in the CVs for salt concentrations of 10 and 100 $\mu \mathrm{M}$. Already, for $1 \mathrm{mM}$ salt concentration, the apparent halfwave potential does not change with flow, so we limit our consideration to this range of electrolyte concentrations.

The redox cycling voltammograms obtained in the nanogap devices are not stretched along the potential axis and exhibit only a systematic shift to lower potentials with increasing flow rates for both the US and DS electrode pairs. This is unlike the UME configuration, which exhibited shifts in opposite directions at the two electrodes. This behavior is expected, as discussed in the theoretical model section, since no ohmic drops are caused by the faradaic processes in this case. The local solution potential is only affected by streaming potentials, which cause a shift that is linear with the flow rate. We therefore estimated $V_{\text {str }}$ by extracting the half-wave potential from the normalized curves and subtracting the value obtained in the absence of flow for every set of measurements

$$
V_{\text {str }}(Q)=E_{1 / 2}(Q)-E_{1 / 2}(0)
$$

The results are shown in Figure 8. The trends are the same as those observed for the UME configuration: the streaming potentials have negative values, depend on the flow rate in an approximately linear fashion, increase with decreasing electrolyte concentration, and have higher magnitudes at the US nanogap compared to the DS device. The magnitude of the effect is however weaker at both for the upstream and downstream nanogap devices in comparison with the UMEs case. We attribute this to the higher conductivity of the solution in this set of experiments. $\mathrm{Ru}\left(\mathrm{NH}_{3}\right)_{6} \mathrm{Cl}_{3}$ is present at a concentration of $100 \mu \mathrm{M}$ and dissociates into ions, $\mathrm{Ru}$ $\left(\mathrm{NH}_{3}\right)_{6} \mathrm{Cl}_{3} \rightarrow \mathrm{Ru}\left(\mathrm{NH}_{3}\right)_{6}^{3+}+3 \mathrm{Cl}^{-}$, significantly impacting the solution conductivity at low salt concentrations. It is also expected that the trivalent cation suppresses the $\zeta$ potential of the channel walls and hence the streaming current. ${ }^{23}$

\section{CONCLUSIONS}

We reported on the behavior of micro- and nanofluidic electrochemical detectors under advective flow control and at low ionic strength. The data indicate that below $1-10 \mathrm{mM}$ 
ionic strength and for typical microfluidic dimensions and flow rates, the solution potential at the electrodes is no longer equal to the reference potential due to streaming potentials in addition to ohmic drops. For negatively charged channel walls, as most commonly encountered in microfluidics, the polarity of the streaming potential is such that it facilitates oxidation reactions and hinders reduction reactions. For oxidation, mechanical energy in the form of fluid flow is converted into (electro) chemical (free) energy and vice versa for reduction. ${ }^{15}$

Our observations further illustrate how streaming potentials in combination with ohmic drops provide a coupling mechanism between electrochemical processes taking place at different electrodes in a fluidic network. Most importantly, the apparent formal potential of a reaction taking place at one electrode can shift to more oxidative or reductive potentials due to reactions taking place at another, nominally independent and spatially remote electrode. This feedback mechanism is particularly relevant in interpreting results from multichannel, miniaturized electroanalytic systems commonly encountered in lab-on-a-chip technology. Fortunately, the complex interplay between electrokinetic effects and electrochemical reactions is semiquantitatively described by a simple circuit model based on lump circuit elements, allowing for rational design and interpretation.

\section{ASSOCIATED CONTENT}

\section{SI Supporting Information}

The Supporting Information is available free of charge at https://pubs.acs.org/doi/10.1021/acs.jpcc.9b08584.

Details of procedure for fitting the equivalent circuit model to data, analysis of the equivalent circuit for parallel flow configuration, details of the simulations, process flow for device fabrication (PDF)

\section{AUTHOR INFORMATION}

\section{Corresponding Author}

Serge G. Lemay - University of Twente, Enschede, The Netherlands; @ orcid.org/0000-0002-0404-3169; Email:s.g.lemay@utwente.nl

\section{Other Authors}

Zinaida A. Kostiuchenko - University of Twente, Enschede, The Netherlands

Jin Z. Cui - University of Twente, Enschede, The Netherlands; ๑ orcid.org/0000-0001-9659-4381

Complete contact information is available at:

https://pubs.acs.org/10.1021/acs.jpcc.9b08584

\section{Notes}

The authors declare no competing financial interest.

\section{ACKNOWLEDGMENTS}

This work was supported financially in part by the European Research Council (ERC) under Project 278801.

\section{REFERENCES}

(1) Bocquet, L.; Charlaix, E. Nanofluidics, from Bulk to Interfaces. Chem. Soc. Rev. 2010, 39, 1073-1095.

(2) Kolthoff, I. M.; Lingane, J. J. The Fundamental Principles and Applications of Electrolysis with the Dropping Mercury Electrode and Heyrovský's Polarographic Method of Chemical Analysis. Chem. Rev. 1939, 24, 1-94.
(3) Bond, A. M.; Fleischmann, M.; Robinson, J. Voltammetric Measurements Using Microelectrodes in Highly Dilute Solutions: Theoretical Considerations. J. Electroanal. Chem. Interfacial Electrochem. 1984, 172, 11-25.

(4) Oldham, K. B.; Zoski, C. G., Chapter 2 Mass Transport to Electrodes. In Comprehensive Chemical Kinetics, Bamford, C. H.; Compton, R. G., Eds.; Elsevier: 1986; Vol. 26, pp 79-143.

(5) Amatore, C.; Deakin, M. R.; Wightman, R. M. Electrochemical Kinetics at Microelectrodes: Part Iv. Electrochemistry in Media of Low Ionic Strength. J. Electroanal. Chem. Interfacial Electrochem. 1987, $225,49-63$.

(6) Norton, J. D.; White, H. S.; Feldberg, S. W. Effect of the Electrical Double Layer on Voltammetry at Microelectrodes. J. Phys. Chem. 1990, 94, 6772-6780.

(7) Ma, C.; Contento, N. M.; Bohn, P. W. Redox Cycling on Recessed Ring-Disk Nanoelectrode Arrays in the Absence of Supporting Electrolyte. J. Am. Chem. Soc. 2014, 136, 7225-7228.

(8) Ma, C.; Xu, W.; Wichert, W. R. A.; Bohn, P. W. Ion Accumulation and Migration Effects on Redox Cycling in Nanopore Electrode Arrays at Low Ionic Strength. ACS Nano 2016, 10, 36583664.

(9) Fan, L.; Liu, Y.; Xiong, J.; White, H. S.; Chen, S. ElectronTransfer Kinetics and Electric Double Layer Effects in NanometerWide Thin-Layer Cells. ACS Nano 2014, 8, 10426-10436.

(10) Xiong, J.; Chen, Q.; Edwards, M. A.; White, H. S. Ion Transport within High Electric Fields in Nanogap Electrochemical Cells. ACS Nano 2015, 9, 8520-8529.

(11) Chen, Q.; McKelvey, K.; Edwards, M. A.; White, H. S. Redox Cycling in Nanogap Electrochemical Cells. The Role of Electrostatics in Determining the Cell Response. J. Phys. Chem. C 2016, 120, 17251-17260.

(12) Mansouri, A.; Bhattacharjee, S.; Kostiuk, L. W. Electrokinetic Energy Conversion by Microchannel Array: Electrical Analogy, Experiments, and Electrode Polarization. J. Phys. Chem. C 2014, $118,24310-24324$.

(13) Chang, C.-C.; Yang, R.-J. Electrokinetic Energy Conversion in Micrometer-Length Nanofluidic Channels. Microfluid. Nanofluid. 2010, 9, 225-241.

(14) Yeh, H. C.; Wang, M.; Chang, C. C.; Yang, R. J. Fundamentals and Modeling of Electrokinetic Transport in Nanochannels. Isr. J. Chem. 2014, 54, 1533-1555.

(15) Dumitrescu, I.; Anand, R. K.; Fosdick, S. E.; Crooks, R. M. Pressure-Driven Bipolar Electrochemistry. J. Am. Chem. Soc. 2011, 133, 4687-4689.

(16) 4 - Electrokinetics and Related Phenomena. In Fundamentals of Interface and Colloid Science, Lyklema, J., Ed.; Academic Press: 1995; Vol. 2, pp 4-1-4-135.

(17) Kirby, B. J.; Hasselbrink, E. F. Zeta Potential of Microfluidic Substrates: 1. Theory, Experimental Techniques, and Effects on Separations. Electrophoresis 2004, 25, 187-202.

(18) Kirby, B. J.; Hasselbrink, E. F. Zeta Potential of Microfluidic Substrates: 2. Data for Polymers. Electrophoresis 2004, 25, 203-213.

(19) Zevenbergen, M. A. G.; Krapf, D.; Zuiddam, M. R.; Lemay, S. G. Mesoscopic Concentration Fluctuations in a Fluidic Nanocavity Detected by Redox Cycling. Nano Lett. 2007, 7, 384-388.

(20) Zevenbergen, M. A. G.; Wolfrum, B. L.; Goluch, E. D.; Singh, P. S.; Lemay, S. G. Fast Electron-Transfer Kinetics Probed in Nanofluidic Channels. J. Am. Chem. Soc. 2009, 131, 11471-11477.

(21) Mathwig, K.; Mampallil, D.; Kang, S.; Lemay, S. G. Electrical Cross-Correlation Spectroscopy: Measuring Picoliter-Per-Minute Flows in Nanochannels. Phys. Rev. Lett. 2012, 109, 118302.

(22) Bruus, H. Acoustofluidics 1: Governing Equations in Microfluidics. Lab Chip 2011, 11, 3742-3751.

(23) van der Heyden, F. H. J.; Stein, D.; Besteman, K.; Lemay, S. G.; Dekker, C. Charge Inversion at High Ionic Strength Studied by Streaming Currents. Phys. Rev. Lett. 2006, 96, 224502. 